\title{
Negation and its impact on the accessibility of text information
}

\author{
BARBARA KAUP \\ Florida State University, Tallahassee, Florida
}

\begin{abstract}
Prior experiments have shown that sentences such as (1) Mary bakes bread but no cookies lead to a reduced accessibility of the concept mentioned in the negated phrase, whereas sentences such as (2) Elizabeth burns the letters but not the photographs do not. In the present article, two explanations for this result are investigated. According to situation model theory (Johnson-Laird, 1983; van Dijk \& Kintsch, 1983), the reason is that the entity mentioned within the negated phrase in (2) is not absent from the described situation. According to discourse representation theory (Kamp, 1981), in contrast, the negation operator in (2) does not reduce the accessibility of the negated concept, because the corresponding discourse referent is not introduced but merely referred to within the operator's scope. In two experiments, participants were presented with narrative texts including negation sentences that either introduced or referred to entities, and that either described a situation in which only the nonnegated or only the negated entity was present. The accessibility of the relevant concepts was measured by means of a probe recognition task. The results support the situation models explanation.
\end{abstract}

Negation is a central means of conveying information in verbal communication. Negative expressions allow a speaker to state explicitly that a particular property does not hold for the state of affairs under consideration. Conveying information about what is not the case is often as useful as, and sometimes even more useful than, conveying information about what $i s$ the case. Negation has been a topic of much research in linguistics and philosophy (for a review, see Horn, 1989), but psycholinguistic studies of the processing of negative expressions are relatively rare. Moreover, most of the existing psycholinguistic studies are concerned with rather global effects of negation-for instance, with the difficulties that people have when they process negative sentences in the context of sentence verification (for a review, see Carpenter \& Just, 1975; see also Reichle, Carpenter, \& Just, 2000) or simple comprehension (e.g., Glenberg, Robertson, Jansen, \& JohnsonGlenberg, 1999) tasks.

For language comprehension research, however, the more "local" effects of negation seem especially interesting. How is negation represented, and does it affect the accessibility of text information? A study by MacDonald

This paper is based on my dissertation, which was conducted with support by the German Research Foundation (DFG) at the Graduate Program in Cognitive Science of Hamburg University. I thank my supervisors Stephanie Kelter and Christopher Habel for their tremendous support in all aspects concerning this work, and Monika Hofmann, Berry Claus, Rolf Zwaan, and two anonymous reviewers for their very valuable comments on earlier versions of this manuscript. I also thank Alexandra Peters and Angela Nachtigall for their help with the data collection. Correspondence should be addressed to B. Kaup, Psychology Department, Florida State University, Tallahassee, FL 32306-1270 (e-mail: kaup@psy.fsu.edu). and Just (1989) is directly relevant to this issue. These researchers presented their participants with sentences such as (1) and, immediately afterward, measured the accessibility of the relevant concepts by means of a probe recognition or word naming task. Probe words that had been mentioned in the negated phrase (cookies) yielded significantly longer response times than did probe words mentioned in the nonnegated phrase (bread).

Almost every weekend, Mary bakes some bread but no cookies for the children.

MacDonald and Just took this result as positive evidence for the hypothesis that readers construct a propositional representation in which the negation operator encapsulates the negated information and thereby reduces the accessibility of this information. A very similar assumption is made by linguistic theories of discourse representation (see, e.g., Kamp, 1981). In order to account for the fact that discourse referents introduced within a negated phrase are not readily available for subsequent pronominal reference, it is assumed that these discourse referents are represented in a special substructure that is not accessible for pronominal reference. Findings by Moxey and colleagues on negative quantifiers (Moxey \& Sanford, 1987; Paterson, Sanford, Moxey, \& Dawydiak, 1998; Sanford, Moxey, \& Paterson, 1996) can also be interpreted as being in line with the hypothesis that negation reduces accessibility. With a variety of different tasks and dependent variables, the authors have shown that sentences with a negative quantifier in the subject position [e.g., (2)] differ from the corresponding sentences with a positive quantifier [e.g., (3)] in how a subsequently encountered plural anaphor (e.g., they) will be interpreted. For positive quantifiers, it is interpreted as referring to the refer- 
ence set of the sentence (i.e., the subset of fans who went to the game), whereas for negative quantifiers, the bias is toward the complement set (i.e., the subset of fans who did not go to the game). Thus, a negative quantifier seems to shift the discourse focus away from the subset explicitly referred to in the sentence, which is very similar to the idea that negation reduces the accessibility of text information. Hence, the standard view on negation seems to be that it functions as an accessibility-reducing operator.

Few of the football fans went to the game.

Many of the football fans went to the game.

A closer look at the materials used by MacDonald and Just (1989), however, reveals that the effect observed by the authors might have an entirely different cause. Maybe it is not the negation operator that is responsible for the accessibility difference, but rather a more situational variable. In the situations described in (1), bread, but not a single cookie, is present. Thus, if the content of the described situation is relevant to the accessibility of concepts during language comprehension, then the effect observed by MacDonald and Just can be attributed to the fact that the nonnegated concept corresponded to an entity present in the described situation, whereas the negated concept did not.

Evaluation of this alternative explanation was the goal of the two experiments reported in Kaup (1997). Participants were presented with narrative texts, each of which contained a negative sentence similar to the ones used by MacDonald and Just (1989). Half of the narratives contained negative sentences such as (1), referring to an action with which an object is usually brought into existence (e.g., baking). The other half contained passages such as (4), with negative sentences that referred to an act of destruction (e.g., burning). With acts of destruction, the negated entity is present in the resulting situation and the nonnegated entity is absent. Thus, for passages of this type, the two explanations lead to different predictions.

Elizabeth tidied up her drawers. She burned the old letters but not the photographs. Afterwards she cleaned up.

Whereas the probe word effect observed by MacDonald and Just was replicated for the creation passages, no accessibility difference was found for the destruction passages. This result raises problems for an account according to which the scope of the negation operator is the only relevant variable. However, the results also raise problems for accounts according to which the only relevant variable is an entity's presence in the described situation. Rather, it seems that both variables play a role with respect to the accessibility of information. For creation passages, the two variables affect accessibility in the same direction, which explains the significant accessibility difference. For destruction passages, in contrast, the two variables counteract each other, which might well be the reason why there was no difference in accessibility following these passages.
What are the implications of these findings for theories of discourse comprehension? The results are hard for minimalistic theories of language comprehension (see, e.g., McKoon \& Ratcliff, 1992) to explain. According to these theories, the comprehension process is completed once a coherent representation of the propositional structure of the text (often called text base) has been established. A situational variable such as being present in the described state of affairs does not affect the text base representation, and according to these theories, a variable of this kind should therefore not have an impact on the accessibility of information. In contrast, the results can be explained by situation model accounts of language comprehension (e.g., Johnson-Laird, 1983; van Dijk \& Kintsch, 1983). According to these accounts, comprehenders construct not only a text base representation, but also a representation of the state of affairs described by the text (situation model). A situation model is a referential representation in which the entities that are present in the current situation are foregrounded and are therefore highly accessible (Anderson, Garrod, \& Sanford, 1983; Carreiras, Carriedo, Alonso, \& Fernández, 1997; Glenberg, Meyer, \& Lindem, 1987; Zwaan, Madden, \& Whitten, 2000). Thus, from the perspective of situation model theory, the results can be explained in terms of two variables that affect different levels of representation. The effect of the negation operator can be attributed to the text base level of representation. In a text base representation, negation is usually considered to be an explicitly represented operator with a whole proposition in its scope (e.g., MacDonald $\&$ Just, 1989). Component concepts of a negated proposition are less accessible than those of a nonnegated proposition simply by being encapsulated by the negation operator. ${ }^{1}$ The impact of the situational content, on the other hand, can be attributed to the representational level of situation models. Entities that are present in the current situation are foregrounded at this representational level and should therefore be more accessible than entities that are not present. In conclusion, the experimental results can be explained by assuming that response times to probe words are a function of their accessibility at the text base level of representation as well as of their accessibility at the representational level of situation models. For acts of creation, the accessibility effects are parallel for the two representational levels, and this is why these passages produce a probe word effect. For acts of destruction, the effects are counteracting, and this is why these passages result in a null effect. This explanation will be called the two-levels-of-representationaccount in that which follows.

However, there is another explanation for the fact that the two types of passages yielded different results. The creation and destruction passages differed with respect to the type of noun phrase (NP) used in the object position. In the creation passages, the object NPs were indefinite (see [1]), whereas in the destruction passages, they were definite (see [4]). This difference plays an important role in what can be considered the standard view of discourse 
representation in linguistics. In that which follows, this standard view will be exemplified by Kamp's (1981) discourse representation theory (DRT). Stated in psychological terms, DRT posits that the processing of NPs gives rise to the construction of discourse referents. Not all discourse referents are inserted into the accessible top structure of the discourse representation. According to DRT, a discourse referent introduced within a negative phrase, for instance, is inserted into a special substructure that is inaccessible for reference resolution. ${ }^{2}$ Thus, according to DRT, a sentence such as Mary bakes bread but no cookies leads to a representation in which bread is represented at the accessible top level, whereas the discourse referent standing for cookies is located in a nonaccessible substructure (see Figure 1A). In contrast, a definite NP does not introduce an entity, but refers to an entity (see also Heim, 1982; van der Sandt, 1992). Usually, the corresponding discourse referent is therefore already part of the representation, and if not, it will be inserted into the accessible top structure. Accordingly, a sentence such as Elizabeth burns the letters but not the photographs leads to a representation in which the letters and the photographs are both represented at the accessible top level (see Figure 1B). Thus, DRT offers an explanation of the results for which the different types of actions mentioned are irrelevant. Rather, the decisive variable is whether the NPs in the negation sentences are indefinite or definite. Only with indefinite NPs is the negated discourse referent located in the nonaccessible substructure, and this is why there is a probe word effect after sentences with indefinite NPs but not after sentences with definite NPs.

It should be pointed out that the distinction between top structure and substructure discourse referents is a distinction between discourse referents that represent entities of the discourse world and those that do not. Of the discourse referents introduced or referred to in (1) and (4), only the

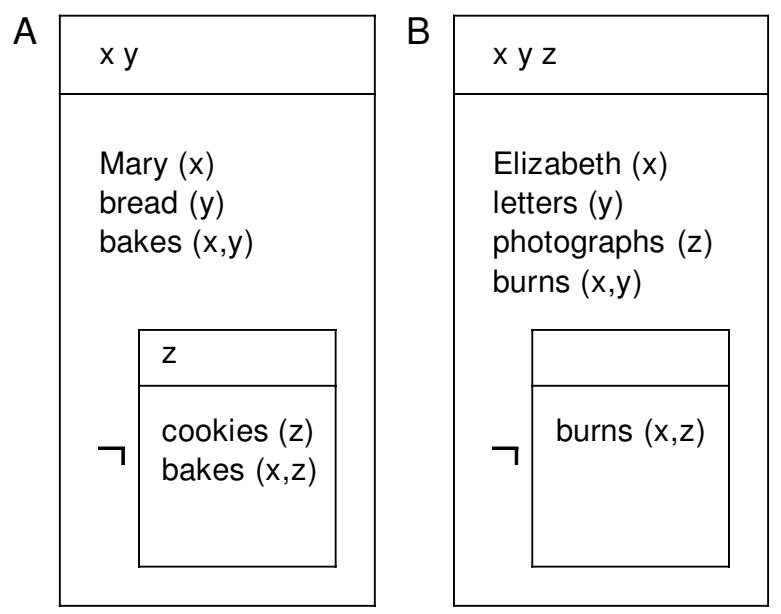

Figure 1. DRT Representation of the sentences (A) Mary bakes bread but no cookies, and (B) Elizabeth burns the letters but not the photographs. one standing for cookies does not represent an entity of the discourse world, and accordingly this is the only discourse referent that is represented in the nonaccessible substructure. ${ }^{3}$ Thus, like situation model theory, DRT posits that language comprehension consists of constructing a referential representation in which tokens standing for entities of the discourse world are kept available. In contrast to situation model theory, however, DRT does not distinguish between entities that are present in the current situation and those that are not. According to DRT, all entities of the discourse world are equally accessible.

The main goal of the present study was to find out which of the two outlined accounts is correct, or, in other words, whether the differential results obtained for sentences such as (1) and (4) are due to the different types of actions mentioned (bringing into the situation vs. taking out of the situation; two-levels account) or to the different types of NPs used in the object position (indefinite vs. definite; DRT-based account). It should be noted, however, that an empirical evaluation of the two accounts is not only relevant to the specific question of why the two sentence types lead to different accessibility patterns, but also to more general issues regarding the nature of the referential representations constructed in discourse comprehension. Results in favor of the two-levels hypothesis would imply that the current situation is foregrounded at this representational level, with present entities being relatively more accessible than absent entities. Results in favor of the DRT-based hypothesis, in contrast, would suggest that the content of the current situation is irrelevant, and that all discourse entities are equally accessible at the referential level of representation.

\section{EXPERIMENT 1}

The goal of Experiment 1 was to investigate the impact of NP type on the probe word effect. Participants were presented with narrative texts containing negation sentences that mentioned acts of creation. In half of the negation sentences, the relevant NPs were indefinite (e.g., [5]), and in the other half they were definite (e.g., [6]).

Sarah is building a chair but not a table.

John is building the castle but not the church. ${ }^{4}$

According to the DRT-based account, negation sentences with indefinite NPs introduce discourse referents and lead to a representation in which only the nonnegated entity is accessible (see Figure 2A), whereas negation sentences with definite NPs refer to discourse referents and lead to a representation in which both entities are accessible (see Figure 2B). If this is correct, the present experiment should produce an interaction of probe word and NP type, such that there would be a probe word effect for the indefinite negation sentences but not for the definite negation sentences.

According to the two-levels account, in contrast, the decisive variable is the type of action mentioned in the 

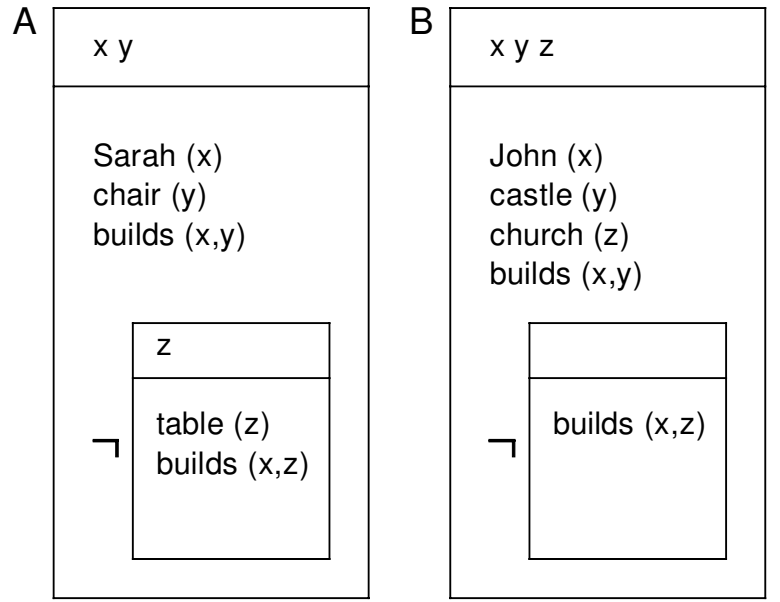

Figure 2. DRT Representation of the sentences (A) Sarah builds a chair but not a table, and (B) John builds the castle but not the church.

negation sentences. In this experiment, all negation sentences mentioned acts of construction, and with these, the nonnegated entities should be more accessible than the negated entities on both representational levels. Thus, if the two-levels account is correct, the present experiment should yield a main effect of probe word that is independent of NP type.

\section{Method}

Participants. Thirty-two Hamburg University students took part in the experiment.

Materials. The materials consisted of 51 German passages, each describing an event. Sixteen of these were experimental passages, and 35 were filler passages. Three passages each belonged together thematically and formed a coherent narrative text.

The experimental passages were constructed according to the following schema (see the examples in Tables 1 and 2): After a short introductory section, the negation sentence followed, being either of the form ...a NOUN 1 , but not $a \mathrm{NOUN}_{2}$ (indefinite NPs in object position), or of the form ... the $\mathrm{NOUN}_{1}$, but not the $\mathrm{NOUN}_{2}$ (definite NPs in object position). The verb of the sentence denoted an action that brings an object into existence (e.g., to bake, to knit, to build). The two nouns named Objects A and B that could each be conceived of as the outcome of the action equally well. For half of the participants, $\operatorname{NOUN}_{1}$ named object A and $\mathrm{NOUN}_{2}$ named object B; for the other participants, the assignment was reversed. The subsequent sentence described the completion of the action without reference to one of the objects. Probe words used in the experimental trials were the nouns $\left(\right.$ NOUN $_{1}$, NOUN $\left._{2}\right)$ of the negation sentences.

Filler passages varied in length and served to obscure the construction principle. For 10 filler trials, probe words were nouns that had been mentioned in the passage, whereas for the remaining 25 filler trials, the probe words had not been mentioned before.

For each text, there were two statements about the events described in the text. These statements were presented at the end of the corresponding text, and participants were asked to decide whether or not the statements were true. Half of the overall 34 statements required a "yes" response, and the other half, a "no" response.

Design and Procedure. The participants were tested individually. Each participant was presented with all 51 passages. Eight of the 16 experimental passages contained indefinite NPs in the nega- tion sentences, and the remaining 8 contained definite NPs. For each participant, the negated noun was the probe in 4 of these two types of passages, and the nonnegated noun was the probe in the remaining 4 passages. The texts and probe words were displayed on a computer monitor (15-in.) in 14- and 28-point fonts, respectively. Text presentation occurred sentence by sentence, self-paced by the participant pressing the space bar. Pressing the bar after reading the final sentence of a passage elicited the presentation of the probe word with a 2.5 -sec delay. The participants decided as quickly and accurately as possible whether or not the word had been mentioned in the text by pressing the "d" or "l" key, respectively. After the end of each text, the two statements were presented, and the participants decided for each statement whether or not it was true by again pressing the appropriate key. The participants were encouraged to read the material carefully for comprehension. The experimental session lasted approximately $60 \mathrm{~min}$.

\section{Results and Discussion}

Error rates and response latencies in the probe recognition task of the experimental passages were submitted to 2 (NP type) $\times 2$ (probe word) analyses of variance (ANOVAs) with repeated measurement on both factors for the by-participants analysis, and repeated measurement on only the last factor for the by-items analysis. The latency analyses were performed on latencies for correct responses only. Latencies that were higher than $5,000 \mathrm{msec}$ were omitted from the analysis, and of the remaining latencies, only those were analyzed that deviated less than two standard deviations from the mean of the participant in the respective condition (this eliminated less than $1 \%$ of the data). The mean latencies and the percentages of errors are displayed in Table 3.

Errors. The average error rate was 7\% for the experimental items. Error rates were significantly higher for the negated nouns than for the nonnegated nouns $\left[F_{1}(1,31)=\right.$ $10.01, M S_{\mathrm{e}}=0.23, p<.01 ; F_{2}(1,14)=8.76, M S_{\mathrm{e}}=1.03$, $p<.05]$. NP type did not affect the error rates (both

Table 1

Sample Passage From Experiment 1 (Indefinite NPs)

\begin{tabular}{ll}
\hline Title & Christmas Preparations \\
Passage 1 (Fill) & This year Sarah had intended to begin \\
& early with her Christmas preparations. \\
& Now it already is November, and she still \\
& does not have any gifts for friends and \\
& relatives. Therefore, she decides to spend \\
& the whole weekend buying and making \\
& gifts for Christmas.
\end{tabular}

Probe SOFA

Passage 2 (Exp.) She knows that her uncle Carl really likes handcrafted things. For quite a while Sarah tried to come up with nice things she could make for him out of wood. Finally she bought some expensive oak lumber and got going.

Negation Sarah is now building a chair but not a table for her uncle.

Completion Sarah works for hours until she finally is finished.

Probe CHAIR/TABLE

Passage 3 (Fill) 
Table 2

Sample Passage From Experiment 1 (Definite NPs)

Title
Passage 1 (Exp.)

Negation

Completion

Probe

Passage 2 (Fill)

Passage 3 (Exp.)

$F_{\mathrm{S}}<1$ ), and there was no probe word $\times$ NP type interaction (both $F_{\mathrm{s}}<1$ ).

Latencies. The pattern of results for the response times was the same as that for the error rates. There was a significant main effect of probe word, with latencies being longer for the negated than for the nonnegated nouns $\left[F_{1}(1,31)=13.62, M S_{\mathrm{e}}=58,860, p<.01 ; F_{2}(1,14)=5.49\right.$, $\left.M S_{\mathrm{e}}=36,715, p<.05\right]$. NP type did not have any impact on the latencies (both $F \mathrm{~S}<1$ ), and more important, there was no interaction between the two variables $\left[F_{1}(1,31)=\right.$ $\left.1.12, M S_{\mathrm{e}}=61,498, p=.30 ; F_{2}<1\right]$.

The results do not support the DRT-based hypothesis. According to this hypothesis, the difference in the relative accessibility of negated and nonnegated nouns after reading passages such as (1) and (4) is due to the fact that the target concepts are mentioned within indefinite NPs in (1), but within definite NPs in (4). If this hypothesis were correct, the present experiment should have yielded an interaction between NP type and probe word. Contrary to this prediction, however, the results showed a significant main effect of probe word and no probe word $\times$ NP type interaction.

The two-levels hypothesis, in contrast, is in line with the results. The finding that passages such as (1) and (4) lead to different accessibility patterns is attributed to the different type of actions mentioned in these passages. According to this account, mentioning acts of creation produces a probe word effect, whereas mentioning acts of destruction does not. All negation sentences in this experiment mentioned acts of creation, and the observations that there was a main effect of probe word and no interaction with NP type converge to support this prediction.

\section{EXPERIMENT 2}

The main goal of Experiment 2 was to investigate the impact of action type (creation/destruction) on the probe word effect within one factorial design. According to the two-levels hypothesis, the type of action mentioned in the negation sentences is the decisive factor for whether or not a probe word effect is obtained, and being able to directly compare the probe word effect for the two action types was therefore desirable. A second goal of Experiment 2 was to investigate whether the order in which the negated and nonnegated phrases were mentioned would play a role as well. In Experiment 1, the nonnegated noun was always mentioned before the negated noun. Thus, the difference in accessibility of the two target nouns was not necessarily due to the scope of the negation marker but might also have been due to the order in which the two nouns were mentioned. According to this alternative interpretation, the probe word effect could not be considered an effect of negation, but must rather be considered a sort of primacy or advantage-of-first-mention effect (Gernsbacher, 1997). To evaluate this alternative interpretation, the negated noun was always mentioned before the nonnegated noun in Experiment 2. Thus, the two types of negation sentences presented in this experiment were of the types illustrated in (7) and (8), respectively.

John does not build the church but the castle.

Peter does not burn the big cupboard but the old bed.

If the two-levels account was correct, this experiment should produce an interaction between probe word and action type. Nonnegated nouns should be more accessible than negated nouns for creation passages but not for destruction passages.

\section{Method}

Participants. Thirty-two Hamburg University students took part in Experiment 2.

Materials. The materials were similar to those of Experiment 1. However, in Experiment 2, half of the experimental items were creation passages and half were destruction passages. Moreover, the relevant NPs were definite in all experimental passages, and the nonnegated entity was always mentioned after the negated entity. Thus, all negation sentences were of the form ...not the NouN $_{l}$ but the Noun $_{2}$. Except for the modifications with respect to the order in which the negated and the nonnegated nouns were mentioned, the creation passages were identical to the passages with definite NPs used in Experiment 1. An example for a destruction passage is given in Table 4.

Design and Procedure. Each participant was presented with all 51 passages. Half of the 16 experimental passages were creation passages; the other half, destruction passages. For each participant, the negated noun was the probe in 4 passages of each of the two types,

Table 3

Mean Latencies (in Milliseconds) of Correct Responses, Standard Deviations, and Percentages of Errors in Experiments 1 and 2

\begin{tabular}{|c|c|c|c|c|c|c|}
\hline \multirow[b]{3}{*}{ NP-Type/Action Type } & \multicolumn{6}{|c|}{ Probe Word } \\
\hline & \multicolumn{3}{|c|}{ Nonnegated Noun } & \multicolumn{3}{|c|}{ Negated Noun } \\
\hline & RT & $S D$ & $\% \mathrm{E}$ & RT & $S D$ & $\% \mathrm{E}$ \\
\hline \multicolumn{7}{|l|}{ Experiment 1} \\
\hline Indefinite/Creation & 1,133 & 361 & 4 & 1,245 & 413 & 11 \\
\hline Definite/Creation & 1,080 & 331 & 3 & 1,285 & 435 & 9 \\
\hline \multicolumn{7}{|l|}{ Experiment 2} \\
\hline Definite/Creation & 1,062 & 327 & 11 & 1,286 & 364 & 12 \\
\hline Definite/Destruction & 1,224 & 357 & 7 & 1,314 & 406 & 12 \\
\hline
\end{tabular}


Table 4

Sample Passage From Experiment 2 (Destruction)

\begin{tabular}{ll}
\hline Title & $\begin{array}{l}\text { Cleaning up the Attic } \\
\text { Passage 1 (Exp.) }\end{array}$ \\
& $\begin{array}{l}\text { Today is Peter's day off from work. Peter has } \\
\text { decided to clean up the attic. For years, old } \\
\text { furniture has been piling up in there. In order } \\
\text { to create some space in the attic, Peter de- } \\
\text { cides to chop up some of the furniture and } \\
\text { burn it in the garden. }\end{array}$ \\
& $\begin{array}{l}\text { Peter burns the old bed but not the big cup- } \\
\text { board. }\end{array}$ \\
Negation & $\begin{array}{l}\text { After coming back from the garden, Peter } \\
\text { goes to the attic and looks around happily. } \\
\text { Completion }\end{array}$ \\
PED/CUPBOARD \\
Passage 2 (Fill) & $\ldots \ldots \ldots \ldots$ \\
Passage 3 (Exp.) & $\ldots \ldots \ldots \ldots$
\end{tabular}

and the nonnegated noun was the probe in the remaining 4 passages. The procedure was identical to that of Experiment 1 .

\section{Results and Discussion}

The data were analyzed in the same way as were the data of Experiment 1. Less than 1\% of the response times were classified as outliers. The mean latencies of the remaining correct responses, and the percentages of errors are displayed in Table 3.

Errors. The average error rate was $11 \%$ for the experimental items. Neither the probe word nor the action type had any influence on the error rates in the probe recognitiontask (main effects, all $F_{\mathrm{S}}<1$; interaction, both $F_{\mathrm{s}}<1$ ).

Latencies. Participants responded significantly faster with creation passages than with destruction passages $\left[F_{1}(1,31)=6.53, M S_{\mathrm{e}}=44,074, p<.05 ; F_{2}(1,14)=5.18\right.$, $\left.M S_{\mathrm{e}}=12,551, p<.05\right]$. No attempt will be made to interpret this main effect specifically, since action type was varied between items and its main effect may therefore have been due to differences in probe word length and/or frequency.

There was also a significant main effect of probe word. Nonnegated nouns were responded to significantly faster than negated nouns $\left[F_{1}(1,31)=17.84, M S_{\mathrm{e}}=44,067, p<\right.$ $\left..01 ; F_{2}(1,14)=12.45, M S_{\mathrm{e}}=16,646, p<.01\right]$. This finding rules out an advantage-of-first-mention explanation of the probe word effect. In this experiment, the negated nouns were always mentioned first and should therefore have lead to relatively short latencies if this explanation had been correct.

The interaction of probe word and action type was not significant, but just missed the .05 level of significance in the by-participants analysis $\left[F_{1}(1,31)=3.91, M S_{\mathrm{e}}=36,296\right.$, $\left.p=.057 ; F_{2}(1,14)=1.85, M S_{\mathrm{e}}=16,640, p=.20\right]$. In line with the predictions, planned comparisons revealed a highly significant probe word effect for the creation passages $\left[F_{1}(1,31)=16.53, M S_{\mathrm{e}}=48,248, p<.01 ; F_{2}(1,14)=\right.$ $\left.11.95, M S_{\mathrm{e}}=16,640, p<.01\right]$ but only a marginal probe word effect for the destruction passages $\left[F_{1}(1,31)=4.05\right.$,
$M S_{\mathrm{e}}=32,114, p=.053 ; F_{2}(1,14)=2.35, M S_{\mathrm{e}}=16,640$, $p=.15]$.

The interaction was not quite as strong as predicted. Therefore, a meta-analysis was conducted in which latencies of previous experiments (reported in Kaup, 1997) were included as well. More specifically, all those latencies from previous experiments were included that were from conditions directly comparable to those of the present experiment. All in all, the meta-analysis comprised $16 \times$ 32 latencies from creation passages $(8 \times 32$ from the present experiment, and $8 \times 32$ from Experiment 1 ), and $16 \times$ 32 latencies from destruction passages $(8 \times 32$ from the present experiment, and $8 \times 32$ from Kaup, 1997, Experiment 2). Given that these latencies could not be analyzed in a repeated measures design, and that variability due to participants' differing absolute latencies therefore would add to the error variance, it seemed necessary to reduce this variability. For each individual latency, first the mean of the latencies of the corresponding participant was subtracted and then the overall mean of all participants' latencies was added. The resulting values were then submitted to a 2 (action type) $\times 2$ (probe word) ANOVA. This analysis revealed a significant main effect of action type $\left[F(1,252)=8.23, M S_{\mathrm{e}}=29,155, p<.001\right]$, a significant main effect of probe word $\left[F(1,252)=36.92, M S_{\mathrm{e}}=\right.$ $29,155, p<.01]$, and most important, a highly significant interaction of the two variables $\left[F(1,252)=5.89, M S_{\mathrm{e}}=\right.$ $29,155, p<.001]$. For both action types, participants responded faster to the nonnegated nouns than to the negated nouns [creation, $t(126)=5.79, p<.001, S E=31.32$; destruction, $t(126)=2.69, p<.01, S E=29.0]$, but as predicted, the corresponding difference was larger for creation passages (nonnegated, $M=1,143 \mathrm{msec}$; negated, $M=$ $1,324 \mathrm{msec}$ ) than for destruction passages (nonnegated, $M=1,256 \mathrm{msec}$; negated, $M=1,334 \mathrm{msec}$ ).

Together with the results of the meta-analysis, the results of Experiment 2 correspond to the predictions of the two-levels account. Although participants generally responded faster to the nonnegated nouns than to the negated nouns, the corresponding latency difference was larger for the creation passages than for the destruction passages. According to the two-levels account, this interaction reflects the fact that the situational absence reinforces the effect of the negation operator for acts of creation but decreases it for acts of destruction.

It should be noted that the interaction of probe word and action type rules out an interference-based explanation of the probe word effect obtained in the previous experiment (cf. Wiley, Mason, \& Myers, 2001). If the comparatively long latencies to negated probes were merely due to participants' having had problems responding with "yes" to a word that was mentioned within a negative phrase, we would have expected to find a main effect of probe word, but no interaction of probe word and action type.

It could be argued that instead of situational content, a more pragmatic variable might have been responsible for the different results obtained for creation and destruction 
passages. There could have been a difference between the passages with respect to what was considered the normal and what was considered the special condition. More specifically, it could be hypothesized that with the specific creation actions used in this experiment, the normal condition was that things were not created, and the special condition was that something was created, whereas for the specific destruction actions, the normal condition was that everything was destroyed and the special condition was that something was left untouched. If this was true, then the negated phrases would also be more noteworthy for the destruction items than for the creation items, and this in turn could account for the finding that the negated entities were relatively more accessible after the reading of destruction items than after the reading of creation items (for a theory of this type, see Sanford \& Garrod, 1998). 5

In order to examine the impact of this pragmatic variable, a new sample of 26 participants was presented with each experimental item up to the sentence prior to the negation sentence. For each item, they were asked to indicate whether they expected the story to continue with a passage telling them (A) what the protagonist constructed/ destroyed, (B) what the protagonist did not construct/ destroy, or (C) both. In addition, they were asked whether they expected the number of objects that the protagonist constructed/destroyed to be (A) smaller, (B) greater, or (C) equal to the number of objects s/he did not construct/ destroy. ${ }^{6}$

The results of this experiment speak against a strong version of the pragmatic hypothesis. If it were true that the objects not destroyed were of major interest for the destruction items, we should have found that participants expected to be informed about these objects with relative high frequency. In contrast, after reading the introduction of the destruction items, Answer B was selected significantly less often than Answer A [ 23 vs. $93, \chi^{2}(1)=$ $42.24, p<.01]$. Furthermore, if it were true that the normal condition was that everything was destroyed, we should have found participants to expect the number of objects destroyed to be greater than the number of objects not destroyed. In contrast, the relative frequency with which participants chose B for the quantity question of destruction passages was not significantly larger than the frequency with which they chose $\mathrm{A}\left[87\right.$ vs. $70, \chi^{2}(1)=$ $1.8, p>.10]$. However, there was some support for a weaker version of the hypothesis. Answer B was selected more often for the destruction items than for the creation items [continuation, 23 vs. $11, \chi^{2}(1)=4.24, p<.05$; quantity, 87 vs. $\left.38, \chi^{2}(1)=19.21, p<.01\right]$. Thus, there does seem to have been a difference between the two item types with respect to expectations about the described state of affairs.

However, post hoc comparisons indicated that the difference in response latencies could not be attributed to this pragmatic difference between the item types. The destruction items that behaved according to the pragmatic hypothesis on the continuation question (i.e., items for which more than half of the participants expected a continuation passage that informed them about the negated entity) showed a numerically larger probe word effect $(119 \mathrm{msec})$ than did the items for which this was not the case $(66 \mathrm{msec})$. The same held for the question regarding quantity. The probe word effect was numerically larger for the items for which more than half of the participants expected the number of objects destroyed to be greater than the number of objects not destroyed $(115 \mathrm{msec})$ than it was for the items for which this was not the case $(84 \mathrm{msec})$.

\section{GENERAL DISCUSSION}

The goal of this study was to obtain more information about how negatives are processed during discourse comprehension. The focus was on the kind of representation constructed during the processing of negative sentences. More specifically, the study addressed the question of why negated nouns are less accessible than nonnegated nouns after the reading of sentences such as (1) but not, or not as much, after the reading of sentences such as (4).

The results of the experiments do not support the DRTbased explanation, according to which the negation operator reduces the accessibility of a discourse referent only if it is introduced within the scope of the operator (as in [1]), but not if it is merely referred to within the scope of the operator (as in [4]). In contradiction to this hypothesis, Experiment 1 yielded a probe word effect independent of whether the discourse referents were introduced or referred to in the negation sentences.

The results support the predictions of the two-levels hypothesis, according to which negation has a strong impact on the accessibility of concepts mentioned in a text if the negated concepts are absent from the described situation (as in [1]), but has a much weaker impact if this is not the case (as in [4]). In accordance with this prediction, passages with negation sentences of the former type produced a stronger probe word effect than did passages with negation sentences of the latter type.

The results have important implications for discourse comprehension research, in that they rule out particular representational theories while providing positive evidence for others. First, the results pose problems for minimalistic theories, according to which the result of comprehension is a propositional text base representation. Contrary to these theories, the results suggest that there is an additional level of representation at which tokens standing for the referents of linguistic expressions rather than propositions constitute the basic representational unit. In this regard, the results are in line with theoretical arguments that central discourse processes (such as reference resolution) cannot be fully explained without one's assuming such a referential level of representation (cf. JohnsonLaird, 1983; Kamp, 1981; van Dijk \& Kintsch, 1983), as well as in line with a growing body of empirical evidence showing that minimalistic theories are insufficient to ac- 
count for discourse comprehension (for a review, see Zwaan \& Radvansky, 1998). Second, and more important, the results also pose problems for some of the theories that do posit a referential level of representation. One such theory is Kamp's (1981) DRT. According to this theory, discourse entities are kept accessible at the referential level of representation, independently of whether or not they are part of the situation described in the text. Contrary to this assumption, the results of the present experiments suggest that the content of the current situation plays an important role for the accessibility of text information. More specifically, the results indicate that entities that are present in this situation are more accessible at the referential level of representation than are entities that are absent from this situation. Thus, the results pose problems not only for Kamp's specific theory, but for all theories that do not distinguish between entities that are present in the described situation and those that are not. All in all, the results can be explained best by situation model accounts of language comprehension, according to which comprehenders construct a referential representation of the described state of affairs in which the current situation is foregrounded.

\section{REFERENCES}

Anderson, A. Garrod, S. C., \& SAnford, A. J. (1983). The accessibility of pronominal antecedents as a function of episode shifts in narrative text. Quarterly Journal of Experimental Psychology, 35A, 427-440.

Carpenter, P. A., \& Just, M. A. (1975). Sentence comprehension: A psycholinguistic processing model of verification. Psychological Review, 82, 45-73.

Carreiras, M., Carriedo, N., Alonso, M. A., \& Fernández, A. (1997). The role of verb tense and verb aspect in the foregrounding of information during reading. Memory \& Cognition, 25, 438-446.

Gernsbacher, M. A. (1997). Two decades of structure building. Discourse Processes, 23, 265-304.

Glenberg, A. M., Meyer, M., \& Lindem, K. (1987). Mental models contribute to foregrounding during text comprehension. Journal of Memory \& Language, 26, 69-83.

Glenberg, A. M., Robertson, D. A., Jansen, J. L., \& JohnsonGLENBERG, M. C. (1999). Not propositions. Journal of Cognitive Systems Research, 1, 19-33.

HeIm, I. R. (1982). The semantics of definite and indefinite noun phrases. Doctoral dissertation, University of Massachusetts.

Horn, L. R. (1989). A natural history of negation. Chicago: University of Chicago Press.

Johnson-Laird, P. N. (1983). Mental models. Cambridge, MA: Harvard University Press.

KAMP, H. (1981). A theory of truth and semantic representation. In J. Groenendijk, T. Janssen, \& M. Stokhof (Eds.), Formal methods in the study of language (Pt. 1, pp. 277-322). Amsterdam: Mathematisch Centrum.

Kamp, H., \& ReYle, U. (1993). From discourse to logic. Dordrecht: Kluwer.

KAUP, B. (1997). The processing of negatives during discourse comprehension. In M. G. Shafto, \& P. Langley (Eds.), Proceedings of the Nineteenth Conference of the Cognitive Science Society (pp. 370-375). Mahwah, NJ: Erlbaum.

MacDonald, M. C., \& Just, M. A. (1989). Changes in activation levels with negation. Journal of Experimental Psychology: Learning, Memory, \& Cognition, 15, 633-642.

McKoon, G., \& Ratcliff, R. (1992). Inference during reading. Psychological Review, 99, 440-466.

MoXeY, L. M., \& SANFoRD, A J. (1987). Quantifiers and focus. Journal of Semantics, 5, 189-206.

Paterson, K. B., Sanford, A. J., Moxey, L. M., \& Dawydiak, E. (1998). Quantifier polarity and referential focus during reading. Journal of Memory \& Language, 39, 290-306.

Reichle, E. D., Carpenter, P. A., \& Just, M. A. (2000). The neural bases of strategy and skill in sentence-picture verification. Cognitive Psychology, 40, 261-295.

SANFord, A. J., \& GARrod, S. C. (1998). The role of scenario mapping in text comprehension. Discourse Processes, 26, 159-190.

SAnford, A. J., \& Moxey, L. M., \& Paterson, K. B. (1996). Attentional focusing with quantifiers in production and comprehension. Memory \& Cognition, 24, 144-155.

VAN DER SANDT, R. (1992). Presupposition projection as anaphora resolution. Journal of Semantics, 9, 333-377.

VAN DiJK, T. A., \& KinTsch, W. (1983). Strategies of discourse comprehension. New York: Academic Press.

Wiley, J., Mason, R. A., \& MYers, J. L. (2001). Accessibility of potential referents following categorical anaphors. Journal of Experimental Psychology: Learning, Memory, \& Cognition, 27, 1239-1249.

ZwaAn, R. A., Madden, C. J., \& Whitten, S. N. (2000). The presence of an event in the narrated situation affects its availability to the comprehender. Memory \& Cognition, 28, 1022-1028.

ZWAAN, R. A., \& RADVANSKY, G. A (1998). Situation models in language comprehension and memory. Psychological Bulletin, 123, 162-185.

\section{NOTES}

1. MacDonald and Just (1989) found the probe word effect with a naming task. Thus, it is unlikely that the prolonged latencies for negated probes are due to interference between "yes" responses and the presence of the negation in the surface structure.

2. This statement is somewhat simplifying. According to DRT, substructures are not either accessible or inaccessible, but rather accessible from certain representational locations only. Thus, although discourse referents represented in a negated substructure are indeed inaccessible for anaphoric reference at the top level of representation, such an anaphoric reference is possible from a substructure embedded within the negated substructure (for a comprehensive overview, see Kamp \& Reyle, 1993).

3 . Note that whether or not something is considered an entity of the discourse world does not depend on its existence in the discourse world, but rather on whether or not it can be referred to by means of a referential expression. Thus, although the letters no longer exist in the world described in (4), they still constitute a discourse entity in this world, as is, for instance, indicated by the fact that they can be referred to anaphorically (e.g., Sarah burned the letters, because reading them always made her sad).

4. Note that the church constitutes a discourse entity, although it does not exist as a physical entity in the discourse world. The context provides an entity (viz., the particular church that is described in the booklet with suggestions on what to build; see Table 2) to which the NP the church refers. The same does not hold for a table in (5), because there is no corresponding physical or nonphysical entity in the discourse world.

5. I would like to thank one of the reviewers for bringing this possibility to my attention.

6. Note that for each story, the context ensures that the set of objects to choose from is finite (e.g., the objects suggested in the booklet, the objects located in the attic). Thus, it is not a priori clear that the number of objects chosen is smaller than the number of objects not chosen.

(Manuscript received April 27, 2000; revision accepted for publication May 2, 2001.) 